\title{
Valoración económica del recurso hídrico de la microcuenca Quillusara en el cantón Celica- Ecuador
}

\section{Economic valuation of water resources of the watershed Quillusara, canton Celica- Ecuador}

\author{
Diana Bravo-Benavides ${ }^{1}$, Romeo Jaramillo ${ }^{2}$, Diana Encalada ${ }^{3}$ \\ ${ }^{1}$ Departamento de Economía - Universidad Técnica Particular de Loja, 110108, 07 3701444, dbbravo@utpl.edu.ec, \\ http://orcid.org/0000-0002-4506-7255 \\ ${ }_{2}^{2}$ Ingeniero Ambiental, Universidad Técnica Particular de Loja, 110108, 073701444. \\ ${ }^{3}$ Departamento de Economía, Universidad Técnica Particular de Loja, 110108,07 3701444, dencalada1@utpl.edu.ec, \\ https://orcid.org/0000-0001-6462-1277.
}

\begin{abstract}
Resumen
$\mathrm{E}^{1}$ 1 presente estudio tiene por objetivo valorar económicamente el recurso hídrico que provee la Microcuenca Quillusara en Celica-Ecuador e identificar los determinantes de la disposición a pagar (DAP) para la conservación del recurso. Para ello se utilizó el método de valoración contingente y un modelo econométrico LOGIT. La obtención de la información se realizó a través de una encuesta aplicada a 374 usuarios del servicio de agua de la ciudad. Se encontró que el 78.6\% de la población encuestada está dispuesta a pagar por la conservación de las fuentes de agua. Además, el estudio reveló una DAP de $0.92 \mathrm{USD} / \mathrm{mes}$, este valor representa un importante insumo para orientar las decisiones de política pública sobre el cuidado y protección de las fuentes de agua en el cantón.
\end{abstract}

Palabras clave: valoración contingente, disposición de pago, agua, modelo Logit.
Rec.: 30.01.2018. Acept.: 14.05.2019.

Publicado el 30 de junio de 2019

\begin{abstract}
The objective of this study is to evaluate 1 economically the water resource provided by the Quillusara Microbasin in Celica-Ecuador and identify the determinants of willingness to pay (WTP) for the conservation of the resource. For this, the contingent valuation method and a LOGIT econometric model were used. The information was obtained through a survey applied to 374 users of the city's water service. We found that $78.6 \%$ of the surveyed population is willing to pay for the conservation of water sources. In addition, the study revealed that the WTP is 0.92 USD/ month, this value represents an important input to guide the public policy decisions on the care and protection of water sources in the canton.
\end{abstract}

Keywords: contingent valuation, willingness to pay, water, Logit model. 


\section{Introducción}

L os bienes y servicios ambientales son vulnerables al sistema económico debido a que no existe un mercado que refleje las preferencias sociales por ellos. La falta de herramientas de valoración limita la adecuada gestión de los recursos que proveen los ecosistemas. Por lo tanto, la necesidad de valorar económicamente estos bienes y servicios nace de la importancia de reconocer su contribución al desarrollo socioeconómico, su grado de agotamiento y deterioro, así como los efectos que generan sobre los niveles de producción, la salud humana y el bienestar social (Gorfinkiel, 1999; Riera et al., 2005).

En el marco del desarrollo sostenible, varios países en desarrollo han adquirido un alto nivel de apoyo político internacional para optimizar la gestión de sus recursos naturales, fundamentalmente el manejo de cuencas hidrográficas y la adopción de políticas relacionadas e integrar el valor económico del servicio ambiental en la toma de decisiones de proyectos de desarrollo (Goldberg, 2007). Este es el reto para mejorar la calidad de vida de la población humana y hacer realmente viable el derecho de uso de las futuras generaciones sobre los recursos ambientales (Rodríguez et al., 2009; Cordero et al., 2008).

Apesar de que Ecuador cuenta con abundantes fuentes de agua, la mala gestión, distribución y uso han afectado la calidad y cantidad del recurso hídrico, constituyéndose en un grave riesgo ambiental, económico y social, con graves consecuencias en las diferentes actividades productivas del país (PNUMA, Municipalidad de Loja y Naturaleza y Cultura Internacional 2007; Núñez, 2011). En este sentido, la valoración económica de los recursos hídricos ejerce un papel preponderante en la gestión de la demanda y en una mejor distribución entre sus varios usos consuntivos (doméstico, agricultura y ganadería, en la industria) y no consuntivos (fuentes de energía, vía de comunicación, ocio) (WWAP, 2009).

Riera et al. (2005) manifiestan que el método de valoración contingente es una de las técnicas que permite estimar el valor de bienes y servicios para los que no existe mercado, con este método se trata de simular un mercado para estimar la máxima cantidad de dinero que el usuario pagaría por un bien si tuviera que comprarlo, como hace con los demás bienes.

El análisis se basa en la disposición a pagar (DAP) por una mejora en un servicio o por evitar su pérdida, valor que comúnmente se explica por características o factores sociodemográficos (FSD) de la muestra encuestada. Actualmente se postula que la DAP depende también de motivos no económicos, en particular, condicionantes personales denominados factores sociopsicológicos (FSP) (Chaves, 2008; Spash et al., 2009;
Zappi, 2011).

La valoración surge como respuesta al creciente deterioro al que se han visto sometidos los ecosistemas, ocasionando con ello el agotamiento y la degradación de los recursos naturales, situación que al mismo tiempo limita la satisfacción de las diferentes necesidades humanas de la zona de influencia.

En este sentido, el Municipio del cantón Celica, reconociendo sus fortalezas y los riesgos a los que está expuesta su población, busca implementar acciones de protección de los bienes y servicios ambientales que los diferentes ecosistemas les brindan, con el fin de promover una mejor calidad de vida para su gente a través de la protección de la biodiversidad.

Con estos antecedentes, el presente estudio estima el valor económico e identifica los determinantes de la disposición a pagar por la conservación del recurso hídrico de la microcuenca Quillusara en el cantón Celica de la provincia de Loja-Ecuador.

\section{Materiales y métodos}

\section{Zona de estudio}

Este trabajo se realizó en el cantón Celica, ubicado en la región sur occidental de la provincia de Loja, a 172 $\mathrm{km}$ de distancia desde la cabecera provincial, con una extensión territorial de $518 \mathrm{~km}^{2}$ y una población total de 14,468 habitantes (INEC, 2010). Orográficamente está circunscrito en las estribaciones de la cordillera occidental andina a 2026 m s. n. m entre el Pircas y el Pucará. Presenta diversos pisos climáticos, variada agricultura y ganadería (Gobierno Autónomo Descentralizado Municipal de Célica, 2011)

Posee una superficie total de 51,859.55 hectáreas,

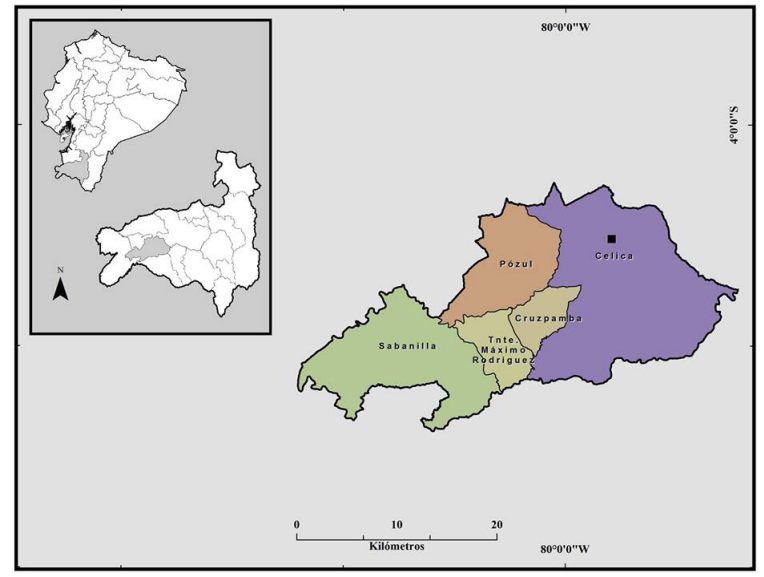

Tomado de: Loaiza, C. (6 de febrero de 2019). Vida Silvestre [Mensaje en un blog]. Christian R. Loaiza S. Recuperado de http://biodiversidadelecuador. blogspot.com/ 
la unidad de cobertura natural dominante es el bosque deciduo piemontano que abarca el $32.95 \%$. La actividad agrícola ocupa una amplia extensión, destacándose el cultivo de maíz con el $23.45 \%$ y el resto de superficie se distribuye entre otros usos productivos (Gobierno Autónomo Descentralizado Municipal de Célica, 2011)

\section{Obtención de información}

Este estudio aplica el Método de Valoración Contingente comúnmente utilizado para aproximar medidas de valoración económica en bienes de no mercado, tales como: la cantidad y calidad del recurso hídrico, servicios de recreación y otros bienes o servicios ambientales. Este método plantea un escenario hipotético que implica cambios en el servicio, a través de una encuesta estructurada (Hanemann, 1994).

En la encuesta se pregunta sobre la disposición a pagar por la conservación del recurso hídrico, la DAP representa el valor económico que los encuestados otorgan a este recurso (Martínez y Dimas, 2007; Webber, 2009; Castro 2011; Aguilar, 2010). La encuesta está dividida en tres secciones: la primera sección, incluye aspectos sociodemográficos; en la segunda sección, se indaga sobre percepción ambiental y en la última sección, se consulta sobre la disposición a pagar para proteger las zonas de interés hídrico.

La muestra se definió con base a los 14,468 habitantes que se benefician del servicio ambiental. Aplicando la fórmula de muestreo aleatorio para poblaciones finitas (Méndez, 2014), se obtuvo una muestra de 374 usuarios.

\section{Modelo de especificación para medir la disposición de pago}

La disposición a pagar por la conservación de la microecuenca Quillusara, se presenta bajo la siguiente condición (Hanemann, 1984).

$$
U(1, Y-A ; S)+\varepsilon_{1} \geq U(0, Y ; S)+\varepsilon_{0}
$$

$U$ se presenta como la función de utilidad indirecta, $Y$ es el ingreso, $A$ es la cantidad monetaria que estaría dispuesto a pagar por el uso del servicio, $S$ es un conjunto de variables socioernnómicas que afectan las preferencias individuales y $\varepsilon_{1 \mathrm{y}} \varepsilon_{0}$ son las variables aleatorias idéntica e independientemente distribuidas con medias cero. La variación de utilidad podría describirse como:

$$
\Delta U=U(1, Y-A ; S)-U(0, Y ; S)+\left(\varepsilon_{1}-\varepsilon_{0}\right)
$$

Para el análisis de la información se seleccionó un modelo de elección binaria Logit, el cual establece que la probabilidad $P_{i}$ de que un individuo acepte un valor monetario $(A)$ está dada por:

$$
P_{i}=F_{n}(\Delta U)=\frac{1}{1+\exp (\Delta U)}=\frac{1}{1+\exp \{-(\alpha-\beta A+\gamma Y+\theta S)\}},
$$

Donde $F_{n}(\Delta U)$ es la función de distribución acumulativa de una variable logística estándar y algunas variables socioeconómicas incluidas en la investigación. Mientras que $\beta, \gamma$ y $\theta$ son coeficientes a estimarse.

Existen tres métodos de calcular el valor de la disposición a pagar, el primero es la "DAP media", cuyo cálculo toma valores de ; el segundo, la "DAP media total", que calcula el valor esperado de la DAP mediante integración numérica en un rango que va de $-\infty y+\infty$; $y$, el tercero, la "DAP media truncada", que también calcula el valor esperado de la DAP mediante la integración numérica cuyo rango va de 0 al valor máximo ofrecido (A) (Duffield y Patterson, 1991). Una vez estimados los parámetros, se utilizará la DAP media, debido a su consistencia teórica y eficiencia estadística.

\section{Resultados}

\section{Aspectos socio-demográficos}

De los 374 encuestados, el $63.9 \%$ son hombres y $36.1 \%$ mujeres. El $67.1 \%$ de los encuestados es originaria de la parroquia Celica, el $17.6 \%$ de la parroquia Sabanilla, el 8\% de la parroquia Cruzpamba, el 4.3 de la parroquia Pózul, el 1.6 de la parroquia Teniente Maximiliano Rodríguez y $1.3 \%$ pertenece a otras parroquias. De estos, el $88.8 \%$ vive en Célica por más de 15 años, $5.6 \%$ de $11-15$ años, $4.8 \%$ de $6-10$ años y el $0.8 \%$ de $1-5$ años.

El $99.5 \%$ es propietario de su vivienda. En el $93.9 \%$ de los casos, la vivienda está habitada por una sola familia, en el $5.6 \%$ por dos familias y en el $0.5 \%$ por 3 o más familias. El $22.2 \%$ de los encuestados se ubica dentro del rango de edades de 15-30 años, el 27.8\% en el de 30-45 años, el $19.8 \%$ en el de $45-55$ años y el $30.2 \%$ en el de mayores de 55 años. El $44.9 \%$ de los usuarios, a quienes se encuestó, estudió la primaria, el 35.6\% completó el bachillerato, el 19\% culminó sus estudios de nivel superior $\mathrm{y}$, tan solo, $0.5 \%$ no tuvo ningún tipo de educación.

Respecto a la actividad económica, el $27.8 \%$ se dedica a la agricultura, $17.1 \%$ son empleados públicos, $16.6 \%$ al comercio, $13.4 \%$ estudiantes secundarios y universitarios, $8.8 \%$ a servicios (restaurantes, hotelería, turismo, transporte), $7.2 \%$ ejercen su actividad como profesionales, $4 \%$ ganaderos, $2.9 \%$ amas de casa, $1.1 \%$ artesanos y $0.5 \%$ jubilados.

En cuanto a dotación de servicios básicos el $76.7 \%$ posee servicio de agua en su domicilio proveniente de la red municipal, $16.3 \%$ obtienen el agua de ríos y quebradas, $6.1 \%$ tiene servicio de agua entubada y $0.8 \%$ obtiene el recurso hídrico de pozos. Respecto al uso del agua, el $100 \%$ la usa para la preparación de alimentos 
e higiene personal. Cabe mencionar que el $30.2 \%$ de la población compra agua en botella y un $28.1 \%$ de ésta adquiere un bidón de 20L semanalmente.

\section{Percepción ambiental}

Los encuestados manifiestan que los problemas que afectan al medio ambiente en el cantón son: la tala de bosques $48.7 \%$, considera que es la basura $19.5 \%$, los incendios forestales $10.2 \%$, la ganadería $7.2 \%$, coinciden entre el incumplimiento de leyes y el uso de agroquímicos $4 \%$, responsabilizan a la minería $2.9 \%$, a la contaminación de vertientes $1.6 \%, \mathrm{CO}_{2} 1.3 \%$ y la cacería $0.5 \%$.

En relación a las consecuencias de los problemas que afectan al medio ambiente, el $29.9 \%$ considera que la perdida de plantas y animales es una de las principales consecuencias, el $27.8 \%$ cree que lo es también la disminución de la cantidad y calidad de agua, el $20.6 \%$ el calentamiento global, $15 \%$ considera la erosión del suelo, $5.9 \%$ la alteración de paisajes y el $0.3 \%$ concuerdan en consecuencias como sequía, falta de sombra y problema a la salud de las personas.

Las principales actividades afectan a la calidad y cantidad de agua que consumen. El 56.1\% riego menciona al riego, para el $30.2 \%$ a la ganadería, para el $5.9 \%$ la minería, el $5.1 \%$ la cría de animales menores, el $1.3 \%$ desconoce, y para el $0.5 \%$ son la piscicultura y el uso de químicos.

Por otra parte, el $45.5 \%$ conoce los programas o actividades que realiza el GAD Municipal para cuidar los bosques y fuentes de agua en el Cantón, señalando principalmente acciones de reforestación; el 24.3\% desconoce qué tipo de gestiones se están realizando; el $20.3 \%$ sobre algunos programas de educación ambiental que han realizado, el $9.6 \%$ mencionó el pago de una tasa ambiental y el $0.3 \%$ sobre acciones de vigilancia.

En cuanto a los actores involucrados y las actividades para proteger las fuentes de agua, más del 94\% conoce que: los incendios causados después de rozar pueden expandirse y quemar grandes áreas, los bosques en la parte alta de las quebradas aseguran la cantidad de agua para consumo, la deforestación causa daños en las nacientes de las quebradas, el ganado contamina las fuentes de agua afectando su calidad. Alrededor del $64 \%$ de los propietarios de las tierras tiene conocimiento sobre los incentivos que pueden recibir por conservar estás áreas y que el pago de una tasa ambiental sirve para cuidar el agua para consumo humano.

Sobre la importancia del bosque para mejorar la cantidad y calidad del agua, el 97.6\% de los encuestados coincide en que los bosques y páramos naturales ayudan a mantener el agua de las quebradas; más del $76 \%$ menciona que la cantidad de agua disponible para consumo es suficiente y de buena calidad. El $64.2 \%$ señala que los propietarios de los terrenos de la microcuenca ayudan a cuidar los bosques.

Con respecto a las instituciones que trabajan por el cuidado del medio ambiente en el cantón Célica, el $88 \%$ de la población encuestada afirmó que quien trabaja con ese propósito es el Gobierno Municipal; 9.6\% se refirió al Ministerio de Ambiente; $1.6 \%$ al Gobierno Provincial; $0.5 \%$ ONGs, refiriéndose principalmente a Naturaleza y Cultura Internacional; y, $0.3 \%$ la Secretaría Nacional del Agua.

\section{Disposición a pagar}

El 78.6\% de la población encuestada está dispuesta a pagar por la conservación de las fuentes de agua. La variable ingreso, que es la variable explicativa más importante de la disposición a pagar, presentó diferencia estadística $(\mathrm{p}<0.01)$ con el signo positivo esperado. Esto indica que la probabilidad de que la disposición a pagar sea "si" aumenta a medida que el ingreso aumenta. El coeficiente estimado de educación registró diferencias estadísticas $(\mathrm{p}<0.05)$, y el signo es positivo como se esperaba (Cuadro 1). Esta variable se expresa en 7 elementos que representan los niveles de educación en el país: sin instrucción, educación básica, bachillerato, superior, tecnología, maestría, otro. Este hallazgo sugiere que la probabilidad de que la disposición a pagar sea "sí" y aumenta a medida que el nivel de educación aumenta.

Los resultados revelan que el $79 \%$ de las observaciones se asignaron correctamente a la DAP prevista en el modelo, sea "sí" o "no", lo que indica un ajuste relativamente bueno a los datos.

Cuadro 1. Valor de uso indirecto (DAP) mediante el modelo Logit

\begin{tabular}{lccc}
\hline Variable & Coeficiente & Valor z & Significancia \\
cons & -3.283673 & -4.48 & 0.000 \\
Y & .0013372 & 2.26 & 0.024 \\
Educ & 1.492689 & 5.94 & 0.000 \\
Número de obs: & & & \\
351 & & \\
LR chi2(2): 53.09 & & \\
Prob > chi2: & \\
0.0000 & \\
Pseudo R2: & \\
0.1458 & \\
Porcentaje de producción correcta: $79.20 \%$ & \\
\hline
\end{tabular}




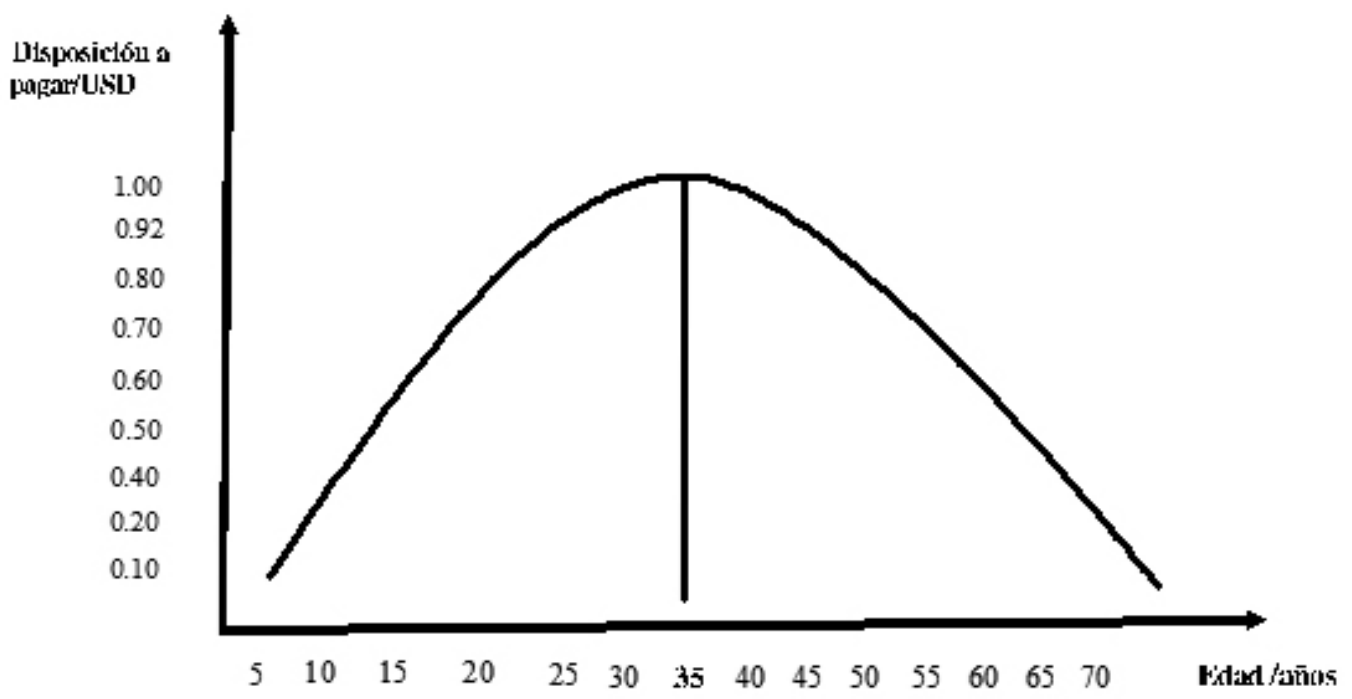

Figura 1. Comportamiento de la edad respecto a la DAP

Después de estimar los parámetros del modelo, utilizando la DAP media, se determinó que valor de uso indirecto de la microcuenca Quillusara fue de 0.92 USD. Este valor representa las unidades monetarias que la población encuestada está dispuesta a pagar mensualmente por la conservación de la microcuenca que abastece de agua al cantón Celica.

La disposición a pagar tiene forma de $\mathrm{U}$ invertida, lo que significa que las personas más jóvenes tienen mayor DAP hasta cierto umbral (35 años), de ahí decrece mientras la edad vaya aumentando (Figura 1).

\section{Discusión}

$\mathrm{A}^{\mathrm{n}}$ pesar de que Celica es un cantón con niveles de ingresos entre medios y bajos, el $79 \%$ de la población está dispuesta aportar económicamente para la conservación de las fuentes de agua de la ciudad, porcentaje superior al de otros estudios realizados previamente en la provincia de Loja (Encalada y Sánchez, 2015; Yaguache, 2009). Por el contrario, a pesar de importancia del recurso, en otras ciudades, como Tulcán y Guaranda, este porcentaje es menor al obtenido en Celica, $59.6 \%$ y $75 \%$, respectivamente (Paspuel y Tobar, 2017; González. et al., 2017).

La media de la DAP fue de 92 centavos de dólar por hogar por mes, este valor es relativamente mayor al obtenido en otras cabeceras cantonales de la provincia de Loja (Encalada y Sánchez, 2015) y relativamente bajo al compararlo con otras ciudades del Ecuador, con características sociodemográficas similares (Paspuel y Tobar, 2017; Rodríguez, et al.,2009). Si se contabiliza la DAP obtenida por todos los hogares de la ciudad, que según el VII Censo de Población y VI de Vivienda (2010) fueron 1,041 dólares se alcanzaría una recaudación promedio anual de aproximadamente 11,500 dólares. Estos valores también sugieren el diseño y aplicación de políticas económicas, por parte del Gobierno Local, para financiar la conservación de la microcuenca y con ello asegurar el bienestar de la población.

En localidades, con mayor población que Celica, como Loja, se ha obtenido una DAP promedio de 5.80/ mes para el financiamiento de planes y proyectos para la protección de fuentes de agua. Este valor representa el $29.5 \%$ del monto de pago promedio mensual (Zapata et al., 2009). En Celica la DAP estimada representa el $30 \%$ de lo que gran parte de los hogares paga mensualmente por el servicio de agua entubada, reflejando la considerable importancia que tiene para la población este recurso.

Respecto a los determinantes de la DAP, en nuestro estudio, al igual que en varios estudios previos (Montes Oca. et al., 2003; Polyzou,et al., 2011; Griffin and Mjelde, 2000; Whittington, et al., 1990), la variable ingreso es estadísticamente significativa y presenta signo positivo. Sin embargo, en otros no ha sido identificada como estadísticamente significativa (Zapata, et al., 2009; Jonhson y Baltonado, 2004; Ortega-Pacheco, et al., 2009).

La educación es también otra variable consistente, es decir, es estadísticamente significativa con signo positivo (Guambo et al., 2016; Kaplowitz et al., 2012), contrario a otros estudios donde presenta signo positivo, pero no significancia estadística (Polyzou et al., 2011; Whittington et al., 1991). 


\section{Conclusiones}

Existe una alta disposición de pago (78.6\%) por la conservación del recurso, esto demuestra que para la población encuestada la conservación de las fuentes de agua es fundamental para asegurar el aprovisionamiento del recurso. El valor económico asignado al recurso mediante la DAP, de $0.92 \mathrm{USD} / \mathrm{mes}$, proporciona la justificación suficiente para que las autoridades establezcan una política impositiva, por ejemplo, una tasa ambiental para proteger la microcuenca y evitar la degradación de los recursos naturales que ésta provee.

Varios parámetros fueron investigados y explorados para identificar los determinantes de la DAP por el cuidado y protección de las fuentes de agua. Específicamente, variables sociodemográficas como el ingreso y el nivel de educación tienen una clara implicación en la DAP, revelando que los ciudadanos con altos niveles de ingreso y escolaridad tienen a atribuir un elevado valor por el mejoramiento de la calidad del agua como resultado de las acciones de conservación de las microcuencas.

\section{Bibliografía}

Aguilar., E. 2010. Valoración económica de los recursos hidrológicos en la microcuenca del Rio Canalí. Tesis de licenciatura, Universidad Autónoma Chapingo, Chapingo, México.

Chaves Esquivel E. 2008. Valoración del agua en la cuenca del río Tempisque: un ejemplo sobre el método de valoración contingente. Uniciencia pp 19-31.

Castro, M. 2011. Una valoración económica del almacenamiento de agua y carbono en los bofedales de los páramos ecuatorianos - la experiencia en OñaNabón-Saraguro-Yacuambi y el Frente Suroccidental de Tungurahua. EcoCiencia / Wetlands International / UTPL / MAE. Quito. pp 17-44

Cordero, D., Moreno-Diaz, A., Kosmus, M., 2008. Manual para el desarrollo de mecanismos de pago/ compensación por servicios ambientales. Equipo Regional de Competencia y Programa GESOREN, GTZ - Ecuador. Deutsche Gesellschaft für Technische Zusammenarbeit (GTZ) GmbH. Quito. Ecuador. pp 23-30.

Cueva, J y L, Chalán. 2010. Cobertura vegetal y uso actual del suelo de la Provincia de Loja. Informe Téenico. Departamento de Sistemas de Información Geográfica de Naturaleza y Cultura Internacional. Gráficas Amazonas. Loja-Ecuador. pp 9-40

Duffield, J., y Patterson, D. 1991. Inference and optimal design for a welfare measure in dichotomous choice contingent valuation. Land Economics, pp 225-239.

Encalada, D., y Sánchez, A. 2015. Climate change and households' willingness to pay for protecting high quality water and its provision in a small basin at Ecuador. Book chapter in Sustainability of Integrated Water Resources Management Water Governance, Climate and Ecohydrology. pp 323-333

Hanemann, W. 1984. Welfare evaluations in contingent valuation experiments with discrete responses. American Journal of Agricultural Economics, pp 332-341.

Hanemann, W. (1994). Valuing the environment through contingent valuation. Journal of Economic Perspectives, pp 19-43

González, M., Monar, G., González, V., Saltos, R., Chávez, L., y Fierro, S. 2017. Valoración económica de los bienes y servicios ambientales en la microcuenca del río Illangama. Revista Ecuatoriana de Investigaciones Agropecuarias, 2(1), 1-7.

Griffin, R., y Mjelde, J. 2000. Valuing Water Supply Reliability. American Journal of Agricultural Economics, 82, 41426.

Goldberg, J. 2007. Valoración económica de las cuencas hidrográficas: Una herramienta para el mejoramiento de la gestión de los recursos hídricos. OEA-Departamento de Desarrollo Sostenible. Guatemala. pp 1- 18.

Gorfinkiel, D. 1999. La valoración económica de los bienes ambientales: una aproximación desde la teoría y la práctica (Tesis de maestría). Universidad de la República. Montevideo, Uruguay.

Gobierno Autónomo Descentralizado Municipal de Célica. 2011. Plan de Desarrollo y O O d e n a mi en t o Territorial. Célica. Loja. Ecuador.

Guambo, A., Arguello, C., Zurita, G., y Romero, J. 2016. El valor económico ambiental de los usuarios del servicio hidrológico de la microcuenca del río Cebadas, provincia de Chimborazo. Sathiri(11), 206-219.

INEC. 2010. Resultados Censo de Población y Vivienda 2010, http://www.inec.gob.ec [Consulta: miércoles, 10 de enero de 2013].

Johnson, N., \& Baltodano, M. 2004. The Economics of Community Watershed Management: Some Evidence from Nicaragua. Ecological Economics, 49(1), 57-71.

Kaplowitz, M. y Lupi, F. \& Arreola, O. 2012. Local Markets for Payments for Environmental Services: Can Small Rural Communities Self-Finance Watershed Protection?. Water Resources Management. 26. 10.1007/s11269-0120097-y.

Martínez, M y Dimas, L. 2007. Valoración económica de los servicios hidrológicos: Subcuenca del Río Teculután. Programa de Comunicaciones WWF Centroamérica. Guatemala. pp 24-28

Méndez L. 2014. Formulación y evaluación de proyectos. Enfoque para emprendedores. (8a. ed). Bogotá. D.C 
Montes de Oca, G., Bateman, I., Tinch, R., y Moffatt, G. (2003). Assessing the Willingness to Pay for Maintained and Improved Water Supplies in Mexico City. Available at: http://www.uea.ac.uk/env/cserge/pub/wp/ecm/ ecm_2003_11.htm.

Núñez, M. 2011. La Cuenca Hidrográfica en la Gestión Integrada de los Recurso Hídricos. Revista Virtual REDESMA. UICN Sur. pp 2-12

Ortega-Pacheco, D., Lupi, F., y Kaplowitz, M. (2009). Payment for environmental services: estimating demand within a tropical watershed. Journal of Natural Resources

Policy Research 1 (2), 189-202.

Paspuel, V., \& Tobar, L. (2017). Valoración económica del servicio ambiental hídrico: para la ciudad de Tulcán. Revista Publicando, 4(11), 135-148.

Polyzou, E., Jones, N., Evangelinos, K., \& Halvadakis, C. (2011). Willingness to pay for drinking water quality improvement. The Journal of Socio-Economics, 40, 7480.

Programa de las Naciones Unidas para el Medio Ambiente PNUMA, Municipalidad de Loja y Naturaleza y Cultura Internacional. 2007. Perspectivas del Medio Ambiente Urbano: GEO Loja. pp 49-76

Riera, P., García, D., Krïstom, B., y Brännlund, R. 2005. Manual de economía ambiental y de los recursos naturales (p. 355). Madrid: Thomson editores y Paraninfo CENGAGE Learning.

Rodríguez, F, Gallarraga, R, Salazar, R, Narváez, N. 2009. Ecuador y los objetivos del milenio: inversiones para la conservación del agua. MAE. pp 23-25

Rodríguez, F., Southgate, D., y Haab, T., (2009). Is better drinking water valued in the Latin American countryside: some evidence from Cotacachi, Ecuador. Water International 34 (3), 325-334.

Spash C., Urama K., Burton R., Kenyon W., Shannon P., Hill G. 2000. Motives behind willingness to pay for improving biodiversity in a water ecosystem: Economics, ethics and social psychology. Ecological Economics, 68: 955-964.

Webber, L. 2009. Diagnóstico y Plan de Monitoreo de la Calidad del Agua en las áreas de Interés Hídrico de los cantones Célica, Pidal, Puyando y Macara. Loja. Ecuador. Naturaleza y Cultura Internacional. pp 2-35.

WWAP. (2009). Water in a changing world: The United Nations world water development report. UNESCO.

WCED. 1987. Report of the World Commission on Environment and Development: Our Common Future. http://www.undocuments.net/wced-ocf.htm http:// desarrollosostenible.wordpress.com/2006/09/27/ informe-brundtland/: Oxford University Press, Oxford.

Whittington, D., Briscoe, J., Mu, X., y Barron, W. 1990. Estimating the Willingness to Pay for Water Services in Developing Countries: A Case Study of the Contingent Valuation in Southern Haiti. Economic Development and Cultural Change, 38(2): 293-311.
Whittington, D., Lauria, D., y Mu, X. 1991. A study of water vending and willingness to pay for water in Onitsha, Nigeria. World Development, 19 (2/3), 179-198.

Yaguache, R. 2009. Análisis Económico del Acuerdo por el Agua: Estudio de Caso Municipio de Celica, Loja, Ecuador. Tesis de maestría. UNAM. México DF.

Zapata, S., Benavides, H., Carpio, C., \& Willis, D. 2009. Economic Value of Basin Protection to Improve the Quality and Reliability of Potable Water Supply: Some Evidence from Ecuador. Water Policy, 14(1), 1-1

Zappi L. 2011. Valoración Contingente: Explorando la disposición a pagar por servicios ambientales declarada por usuarios de la Reserva Nacional Lago Peñuelas. Tesis de maestría. Universidad de Chile. 